\title{
Fairness für weibliche Wissenschaftler und Ärzte - Lehren vom Massachusetts Institute of Technology (MIT)
}

\author{
M. A. Zuber \\ Institut für Klinische Immunologie und Transfusionsmedizin (Direktor: Prof. Dr. F. Emmrich) der Universität Leipzig
}

Klinische Forschung in Deutschland ist bei Medizinern und Naturwissenschaftlern gleichermaßen durch das Fehlen von Frauen in Führungs- und Leitungspositionen gekennzeichnet. Das Phänomen der Chancenungleichheit zwischen Frauen und Männern oder Frauendiskriminierung in der Wissenschaft ist dabei nicht auf Deutschland beschränkt, sondern in vielen Ländern festzustellen. In den Vereinigten Staaten von Amerika ist die Situation für Frauen im allgemeinen günstiger als in Deutschland, aber auch dort ist man von einer praktizierten Gleichberechtigung weit entfernt.

Eine im Jahre 1993 durchgeführte Studie (4) der National Science Foundation stellte fest, dass das durchschnittliche Einkommen weiblicher Naturwissenschaftlerinnen verschiedener Fachrichtungen nur 78\% dessen der Männer beträgt. Frauen in allen Rängen an Colleges und Universitäten haben niedrigere Einkommen verglichen mit Männern gleicher Qualifizierung und Leistung. Frauen haben weniger häufig unbefristete Arbeitsverträge, und sie arbeiten an weniger angesehenen Institutionen. Sie werden langsamer befördert und sind unter den niederen Rängen der Beschäftigten und unter Arbeitslosen überrepräsentiert bei gleichzeitiger Unterrepräsentation in Führungspositionen. Die Ungleichbehandlung von Frauen und Männern kommt mit zunehmendem Karriereverlauf immer stärker zur Geltung.

Das Massachusetts Institute of Technology (MIT) veröffentlichte in der März-Ausgabe seines MIT Faculty Newsletter (1) des Jahres 1999 einen bemerkenswerten Bericht, der beschreibt, wie die dortigen weiblichen Fakultätsmitglieder diesbezügliche Probleme entdeckten, angingen und die Verwaltung von der Notwendigkeit substanzieller Veränderungen überzeugen konnten.

\section{Bericht über eine Initiative zur Besserstellung von Frauen am Massachusetts Institute of Technology (MIT) \\ Bildung eines Komitees über die Belange weiblicher Professoren}

Die Biologie-Professorin Nancy Hopkins beantragte im Jahre 1994 zusätzlichen Laborraum, der ihr nicht bewilligt wurde. Verärgert maß sie die Laborräume von weiblichen und männlichen Wissenschaftlern in der Abteilung für Biologie aus und stellte fest, dass Männer doppelt soviel Laborraum beanspruchten wie Frauen.

Die Professorin redete mit zwei anderen weiblichen Fakultätsmitgliedern über diese Problematik, wodurch eine Diskussion über die Qualität ihrer aller beruflicher Arbeit am MIT in Gang gesetzt wurde. Die Frauen stellten im Verlaufe ihres beruflichen Lebens fest, dass sich ihr Karriereverlauf signifikant von dem ihrer männlichen Kollegen unterschieden hatte. Interessanterweise hatten sie bisher das Thema nie untereinander diskutiert. Am Anfang ihres Gedankenaustausches waren sie zunächst unsicher

Dtsch. Med. Wschr. 2001; 126: 59-62

(c) Georg Thieme Verlag Stuttgart . New York darüber, ob ihre Erlebnisse spezifisch und ihre Wahrnehmung akurat war. Allerdings merkten sie schnell, dass ihren Erfahrungen ein Muster zugrundelag. Neugierig geworden, starteten sie eine Umfrage unter den 15 weiblichen Professoren, welche nur $7,2 \%$ der gesamten Professorenschaft ausmachten. Wie eine Nachfrage im Planungsbüro des MIT zutage brachte, hatte sich dieser geringe Frauenanteil seit mehr als einem Jahrzehnt nicht verändert.

Die drei Frauen befragten 12 weitere Professorinnen von der naturwissenschaftlichen und zwei Frauen von der Fakultät für Ingenieurwesen, welche ihre Erfahrungen teilten. Diskussionen mit Frauen anderer Fakultäten ergaben, dass die geschlechtsbedingten Benachteiligungen das berufliche Leben der meisten negativ beeinflusst hatte unabhängig von Institution und Fachgebiet.

Im August 1994 schließlich überbrachten die 17 Professorinnen ein Schreiben an den Dekan Birgeneau, in welchem sie vorschlugen, eine Initiative zwecks Verbesserung des Status der weiblichen Fakultätsmitglieder zu gründen.

"Dieser Vorschlag dokumentiert unsere grosse Sorge über die geringe Anzahl von weiblichen Mitarbeiterinnen mit Dauerarbeitsverträgen, über ihren Status und ihre Behandlung.

Wir glauben, dass bei der Einstellung jüngerer Fakultätsmitglieder eine diskriminatorische Haltung gegenüber Frauen zur Geltung kommt. Diese von Frauen und Männern gleichermassen an den Tag gelegte Einstellung erfolgt zum überwiegenden Teil unbewusst. Es ist oft schwierig, Diskriminierung als Faktor festzustellen, da immer irgendwelche Umstände zur Erklärung herangezogen werden können..... Daher besteht die Notwendigkeit, Vorsichtsmassnahmen zu ergreifen, um die einzelnen Erfahrungen, die zusammengenommen Geschlechterdiskriminierung bedeuten, zu verhindern, aufzudecken und sofort zu korrigieren.....

Ungleiche Geschlechterbehandlung beeinträchtigt die Lebensqualität der betroffenen Frauen und bewirkt damit, daß die dienstälteren benachteiligten Frauen negative Leitfiguren für jüngere Frauen darstellen.....

Das Kernproblem besteht darin, dass gleiches Talent und gleiche Leistung als ungleich betrachtet werden, wenn sie mit den Augen des Vorurteils gesehen werden. Es besteht der Eindruck, dass Ungleichheiten bezüglich Raum, Gehalt, Verleihung von Auszeichnungen und anderen Formen der Anerkennung zwischen Frauen und Männern vorhanden sind. In vielen Departments besteht für Frauen eine glass ceiling, eine unsichtbare Barriere auf dem Karriereweg nach oben.

Die Aufgabe des Komitees sollte sein, Raumverteilung, Gehälter, Lehrverpflichtungen zu überwachen, um Fairness und Gerechtigkeit zwischen Frauen und Männern herzustellen. Werden Ungleichbehandlungen festgestellt, sollte es einen standardisierten Mechanismus geben, unverzüglich Massnahmen folgen zu lassen, um diese Ungleichbehandlung zu korrigieren." 
Diese Forderung nach der Etablierung eines Komitees kostete die weiblichen Fakultätsmitgliedern einige Anstrengung und Überwindung, da sie fürchteten, als Unruhestifterinnen und Nörglerinnen gebrandmarkt zu werden.

Als Reaktion auf die Forderung der Frauen stellte der Dekan selbst schnell Nachforschungen an und erkannte, dass tatsächlich ein ernsthaftes Problem bestand. Daher war er von der Richtigkeit und Notwendigkeit der Initiative überzeugt und unterstützte das Anliegen der Frauen. Auch abwehrende Argumente der Verwaltung, dass das MIT eben eine maskuline Kultur aufweise, gegen die man nun mal nichts unternehmen könne, drangen nicht durch. Mit der Unterstützung des Präsidenten Charles Vest gelang es dem Dekan Robert Birgeneau die Leiter der einzelnen Departments zur Etablierung dieses Komitees zu gewinnen.

\section{Zusammensetzung und Arbeitsweise des Komitees}

Das Komitee setzte sich schließlich aus je einer Professorin von jeder der sechs Abteilungen in den Naturwissenschaften außer Mathematik zusammen, wo es keine Frau in dieser Position gab, und drei älteren männlichen Fakultätsmitgliedern, welche entweder Abteilungsleiter waren oder gewesen waren, was wegen des damit verbundenen administrativen Wissens und der Erfahrung wichtig erschien.

Das Komitee sammelte in der Folgezeit Informationen von Professorinnen und Abteilungsleitern, die teils aus Daten, teils aus Befragungen bestanden. Dabei handelte es sich um Angaben bezüglich Frauen versus Männer betreffend das Gehalt, verliehene Preise, Drittmittel, Lehrverpflichtungen, Mitgliedschaften in internen und externen Gremien und Informationen über Studenten. Alle Frauen mit unbefristeten Arbeitsverträgen und alle $\mathrm{Ab}$ teilungsleiter wurden zudem persönlich befragt.

Jede einzelne Information wurde allen Professorinnen zugängig gemacht. Es hatte sich herausgestellt, dass die dominierendste Erfahrung der Frauen bisher gewesen ist, dass sie von wichtigen Informationen und Angelegenheiten ausgeschlossen waren und daher auch nicht mitreden konnten.

\section{Ergebnisse des Komitees}

Es ergab sich, dass in einigen Abteilungen Frauen und Männer nahezu gleichen Raum und Anerkennung erhielten, in anderen Abteilungen jedoch große Unterschiede bestanden. Obwohl die Gehälter mit Ausnahme der durch Drittmittel erzielten vertraulich und dem Komitee nicht zugängig gemacht wurden, konnten auch hier durch zufällig bekannt gewordene Zahlen Ungleichheiten festgestellt und korrigiert werden.

Im Rahmen der Faktenermittlung wurde auch der Prozentsatz von Frauen und Männern auf den verschiedenen Stufen der Ausund Weiterbildung vom Studenten bis zum Professor ermittelt und festgestellt, dass auf jeder Stufe der prozentuale Anteil der Frauen abnahm. So betrug, um ein Beispiel zu nennen, der Frauenanteil in der Biologie bei den Studenten 50,9\%, bei den Doktoranden $46,1 \%$, bei den wissenschaftlichen Mitarbeitern 32,1\% und bei den Fakultätsmitgliedern $14,3 \%$. Der Prozentsatz der weiblichen Mitglieder der Fakultät war seit mehr als 10 Jahren auf dem gleichen niedrigen Niveau geblieben, und es gab keinerlei Anzeichen für eine in naher Zukunft eintretende Änderung.

Das Komitee befasste sich auch mit den Eindrücken der Frauen bezüglich ihres eigenen Status und dem ihrer Kolleginnen. Eine
Erfahrung, die Frauen aus allen Abteilungen gleichermassen gemacht hatten, war die, dass Frauen unsichtbar und bedeutungslos waren. Sie nahmen keine wichtigen und Gehör findenden Positionen ein. Sie waren von wichtigen Angelegenheiten ausgeschlossen und an den Rand des Geschehens gedrängt. Mit zunehmendem Verlauf ihrer Karriere fanden sie ihre Tätigkeit schwieriger und weniger zufriedenstellend. Marginalisierung und Bedeutungslosigkeit waren die hauptsächlichen negativen Punkte. Im Gegensatz hierzu hatten jüngere Frauen ganz andere Eindrücke. Sie fühlten sich beachtet und von ihren Abteilungen unterstützt. In ihrer Lebensaltersstufe war ihre größte Sorge die praktische Vereinbarkeit von Familie und Beruf.

Diese unterschiedliche Meinung über den Einfluss ihres weiblichen Geschlechtes auf ihre Tätigkeit zwischen jüngeren und in der Karriere fortgeschrittenen Frauen stellt einen wichtigen Faktor dar. Jede Generation junger Frauen einschließlich der jetzigen Professorinnen, glaubten zu Beginn ihrer Karriere, dass die Geschlechterfrage in der vorangegangenen Generation gelöst worden sei und ihre Karriere nicht beeinflussen werde. Im Verlaufe der Jahre wurden ihnen dann langsam zunehmend die Augen geöffnet über die wahren Machtverhältnisse zwischen den Geschlechtern und sie mussten schließlich feststellen, dass sie einen viel zu hohen persönlichen und beruflichen Preis für das Erreichte zahlen mussten verglichen mit Männern. Auch diese Professorinnen hatten sich von ihren Abteilungen unterstützt und gleichberechtigt gefühlt, als sie jung waren und am Anfang ihrer beruflichen Karriere gestanden hatten.

\section{Empfehlungen des Komitees}

Das Komitee empfahl der Verwaltung einen Katalog von Maßnahmen, um die bestehenden Unzulänglichkeiten zu beseitigen (Tab.1), der Modellcharakter trägt.

\section{Erfolge des Dekans, den Status und die Gleichbehandlung von Professorinnen zu verbessern}

Nachdem das Komitee im Sommer 1995 einen Zwischenbericht erstellt hatte, unternahm der Dekan unverzüglich Schritte, Ungleichbehandlungen zu beseitigen. Frauen wurden in wichtige Angelegenheiten der Departments einbezogen, der Prozentsatz neu eingestellter Frauen auf allen Ebenen vergrößert. Diese Maßnahmen erwiesen sich als von größtem Wert für die Lebens- und Berufsqualität der Frauen. Eine Professorin beschrieb das Ergebnis der Zusammenarbeit so, dass während eines Jahres ein größerer Fortschritt für die Frauen erzielt worden war als im gesamten vergangenen Jahrzehnt. Eine andere Frau erzählte, dass sie seit langer Zeit unzufrieden gewesen war. Sie dachte, dies sei eben der Preis, den man dafür zahlen müsse, eine Wissenschaftlerin an einem Elite-Institut zu sein. Nach der Etablierung des Komitees und seiner Erfolge begann plötzlich ihre Forschung aufzublühen, ihre Drittmittel verdreifachten sich in kürzester Zeit. Sie schätzt nun jeden Aspekt ihrer beruflichen Tätigkeit.

Als ein Resultat der Aktivitäten des Komitees kann auch gewertet werden, dass sich in der Folgezeit der Prozentsatz der am Institut beschäftigten Frauen verbesserte. Während im Jahre 199422 Frauen und 252 Männer beschäftigt waren, waren es im Jahre 199931 Frauen und 235 Männer, wobei in diesem Jahr mit $11,65 \%$ erstmals die Marke von $10 \%$ überschritten wurde. Alleine im Jahr 1999 stieg der Prozentsatz der unbefristet beschäftigten weiblichen Wissenschaftlerinnen um mehr als $40 \%$ an, nämlich von 22 auf 31. 
Tab.1 Empfehlungen des Komitees über weibliche Professoren an der naturwissenschaftlichen Fakultät des Massachusetts Institute of Technology (MIT) an die Verwaltung (1).

a. Empfehlungen zur Gewährleistung und Verbesserung der Gleichstellung von weiblichen Fakultätsmitgliedern

1. Ein Komitee zu Frauenfragen sollte dauerhaft etabliert werden. Seine Aufgaben sind

- Sicherstellung der Kommunikation zwischen Abteilungsleitern und weiblichen Fakultätsmitgliedern

- Datenerhebung zur Gleichstellung der Geschlechter, welche in einem jährlich abzufassenden Bericht dem gesamten MIT zugänglich gemacht werden sollen

- Anheben des allgemeinen Bewusstseins über die Notwendigkeit der Gleichstellung von Frau und Mann

2. Frauen sollen einflussreiche Positionen in den Abteilungen und der Verwaltung einnehmen, außerdem als Vorsitzende und Mitglieder von wichtigen Gremien tätig sein und bei der Auswahl von Verwaltungspersonal mitwirken

3. Überprüfung des Vergütungssystems und Verteilung der Ressourcen im Sinne der Geschlechtergleichstellung

4. Entlassung von (Verwaltungs) personal, das frauendiskriminatorische Praktiken anwendet oder duldet. Sofortige Korrektur, wenn Benachteiligungen von Frauen bekannt werden

5. Verhinderung von Isolierung und schleichender Marginalisierung von Frauen, was besonders nach Erteilung eines unbefristeten Arbeitsvertrages eine häufige Praxis ist

b. Empfehlungen zur Verbesserung des beruflichen Lebens von jüngeren weiblichen Fakultätsmitgliedern

1. Maßnahmen zur Integration und Verhinderung von Isolation von jüngeren weiblichen Fakultätsmitgliedern

2. Regelung des Themas der Familienplanung

3. Vereinheitlichung des Schwangerschaftsurlaubes und eine angepasste Regelung des Zeitraumes zur Erreichung von tenure (unbefristeter Arbeitsvertrag)

4. Ergreifung von Maßnahmen zur Abschaffung des Vorurteiles, dass Frauen mit Kindern weniger leistungsfähig seien als Frauen ohne Kinder und/oder Männer

c. Empfehlungen zur Vergrößerung des Anteils weiblicher Professoren

1. Abteilungsleiter werden verpflichtet, Frauen in Berufungskommissionen aufzunehmen

2. Anhebung des Anteils der Rekrutierung von Frauen auch innerhalb des MIT bei neuen Fakultätsmitgliedern

3. Die Abteilungsleiter sollen jährlich daran erinnert werden, eine genügende Anzahl von Frauen von außerhalb des MIT zu rekrutieren

4. Adäquate Diskussion und Lösung des Familienkonfliktes jüngerer Frauen und Anbieten von geeigneten Lösungen unter Einbeziehung des Rates der älteren weiblichen Fakultätsmitgliedern, um mehr Studentinnen und Postdocs für eine akademische Laufbahn am MIT zu gewinnen

\section{Ursachen der Ungleichbehandlung von Frauen und Männern - Geschlechterdiskriminierung 1999}

Führt man sich den geringen Frauenanteil unter den Fakultätsmitgliedern vor Augen, so würde man annehmen, dass diese gut bezahlt würden und Vergünstigungen in Anspruch nehmen könnten. Stattdessen waren sie unterbezahlt, hatten vermindert Zugang zu den Ressourcen des MIT und waren von jeglichen Machtpositionen ausgeschlossen. Wie konnte es zu solch einer erstaunlichen Situation kommen?

Die mögliche Antwort, dass diese Frauen schlecht behandelt wurden, weil sie nicht gut genug waren, ist falsch. Es handelte sich um außergewöhnliche Frauen, die trotz Benachteiligung Erstaunliches geleistet haben. $40 \%$ der Professorinnen gehören der National Academy of Sciences und/oder der American Academy of Arts and Sciences an. Diese Frauen lehnten auch eine Quotenregelung aus Qualitätsgründen ab.

Das Komitee stellte fest, dass eine wesentliche Erklärung trotz der komplexen Natur der Gründe für die Diskriminierung in einer kollektiven Unwissenheit zu suchen ist. Das, was den Professorinnen am MIT widerfuhr, ist genau das, was Diskriminierung heute, im Zeitalter der juristischen Gleichberechtigung von Frau und Mann ausmacht. Es definiert Diskriminierung in dem Zeitabschnitt 1970 bis heute. Für die Frauen war es sehr schwer festzustellen, zu realisieren und zu formulieren, was passierte, was Diskriminierung war, weil es auf den ersten Blick eben nicht wie Diskriminierung aussah, da es in subtiler, schleichender, persistierender und manipulativer Weise geschah. Alle beteiligten Frauen waren der Meinung, das Problem der Diskriminierung sei mit den Grundrechten und Quotenmaßnahmen gelöst worden. Ungleichbehandlung der Geschlechter verläuft heute in verschiedenen Formen und ist oft schwer zu erkennen. Für ein einzelnes, als Diskriminierung erfahrenes Erlebnis gibt es immer auch irgendeine andere Erklärung als Diskriminierung. Erst durch die Zusammenarbeit aller Beteiligten, des Dekans und aller Frauen, durch Erfahrungsaustausch und systematisches Analysieren der Daten war die Tatsache der Diskriminierung unwiderlegbar. Das Komitee identifizierte hiermit Formen und Muster, denen Geschlechterdiskriminierung heute folgen: Diskriminierung besteht aus einem Muster von wirkungsvollen, aber unerkannten Annahmen und Einstellungen, welches zusammengenommen in systematischer Weise gegen Frauen arbeitet bei gleichzeitiger Vorgabe eines guten Willens, Gleichberechtigung walten zu lassen.

\section{Lösungsvorschläge mit Langzeitwirkung, Gegenmaßnahmen für 1999}

Es handelte sich bei diesem Projekt um eine außergewöhnliche Initiative engagierter Frauen und eines wohlwollenden Dekans bei einem allerdings nur geringen Prozentsatz von Verwaltungsleuten, die die Sache wirklich verstanden hatten und von ihrer Notwendigkeit überzeugt waren. Die Zahl der Frauen am MIT ist trotz der erzielten Fortschritte nach wie vor gering. Es wurde ausgerechnet, dass es 40 Jahre dauern würde, bis trotz verstärkter Einstellung von Frauen $40 \%$ der Professoren weiblich sind.

Sicherstellung der Gleichbehandlung von Frauen und Männern in allen Fakultäten des MIT, Verbesserung der Lebensqualität für jüngere Mitarbeiterinnen und Ausdehnung der Initiative, um Minderheiten am MIT zu stärken, wurden als wichtige Langzeitziele herausgearbeitet.

Das Komitee hält zur Langzeitsicherung ihrer kleinen Erfolge die bewusste und konsequente Umsetzung ihrer an die Verwaltung abgegebenen Empfehlungen und deren Übertragung auf alle Fakultäten des MIT für unerlässlich, da es vermutlich viele Jahre 
dauern wird, bis Geschlechtergleichheit nicht nur vor dem Gesetz, sondern auch im praktischen Leben Realität geworden ist.

\section{Kommentar}

In Deutschland ist man sich seit Beginn der 80er-Jahre einer Unterrepräsentation von Frauen in leitenden Positionen bewusst. Daher etablierte man Frauenförderrichtlinien und Frauenbeauftragte, stellte finanzielle Mittel für Habilitationsstipendien im Rahmen der Hochschulsonderprogramme zur Verfügung, und im Jahre 2001 wird ein ehrgeiziges Programm (2) des Bundesministeriums für Bildung, Wissenschaft, Forschung und Technologie, das zum Ziele hat, den Frauenanteil unter Professoren bis zum Jahre 2005 auf 20\% anzuheben, beginnen. Betrachtet man auf der anderen Seite die Erfolge dieser Maßnahmen, so stellt man für das Fachgebiet Humanmedizin fest, daß der Frauenanteil unter Professoren von 4,4\% im Jahre 1980 auf 6,1\% im Jahre 1997 (5) gestiegen ist. Diese Steigerung ist nicht etwa auf oben genannte Fördermaßnahmen zurückzuführen, sondern ausschließlich auf den etwas höheren Frauenanteil unter Professoren in den 1989 hinzugekommenen neuen Bundesländern (5).

Die hier vorgestellte Studie des MIT belegt, dass Benachteiligung von Frauen heute stattfindet und dokumentiert, daß Diskriminierung unmerklich, subtil, durch Aufaddieren kleiner, kaum merkbarer Benachteiligungen verläuft. Die Studie liefert auch Argumente dafür, warum es nicht ausreicht, lediglich Geld für Förderprogramme bereitzustellen, sondern dass ein Bewußtseinswandel bei den Menschen folgen muss. Das System kann nur durch strikte Maßnahmen geändert und dann fixiert werden, Herz und Geist folgen später durch Gewöhnung und Einsicht nach.

Diskriminierung von Frauen ist auf der einen Seite schwer wirklich zu belegen und auf der anderen Seite so schwer zu bekämpfen, weil sie auf ein unbewusstes Denken zurückzuführen sind, welches Frauen und Männer im Verlaufe ihrer Kindheit übernommen haben und heute nicht mehr zeitgemäß ist. So tendieren sowohl Frauen als auch Männer dazu, Frauen unterzubewerten und Männer überzubewerten. Hierbei handelt es sich um tief verinnerlichte unbewusste Verhaltensweisen, die noch nicht einmal den Weg in das Bewußtsein der öffentlichen Meinung gefunden haben. So hat erst kürzlich Virginia Valian, eine Soziologin und Psychologin vom Hunter Collegés Graduate Studies in ihrem Buch "Why So Slow? The Advancement of Women" (6) eine Erklärung dargelegt, warum Frauen nach wie vor ständig Nachteile erleben. Frauen und Männer haben eine verinnerlichte Geschlechtervorstellung, die nicht mehr zeitgemäß ist und einer Korrektur bedarf. Valian zitiert ein Laborexperiment, bei dem Studenten die Größe eines Mannes und einer Frau auf einer Fotografie angeben sollten, wobei sich auf dem Foto auch als Referenzgegenstand ein Pult befand. Die Studenten schätzen die Männer als größer und die Frauen als kleiner ein als sie tatsächlich waren wegen des Geschlechterschemas, dass Männer größer als Frauen sind. Gleichermaßen assoziieren Frauen und Männer mit Wissenschaftlern Männer, weil eben historisch betrachtet viele Wissenschaftler Männer waren. Frauen werden im täglichen Leben ständig unterschätzt, während Männer überschätzt werden. Frauen werden weniger ernst genommen, wenn sie in Gruppen sprechen als Männer. Der Diskussionsbeitrag einer Frau zu einem Vortrag wird vom Redner weniger ernst genommen als der eines Mannes. Handeln nach diesen Geschlechter-Schemata erfolgt größtenteils unbewusst. Die Realität kann daher nur geändert werden, wenn diese Schemata bewusst gemacht werden und mit großen Anstrengungen und rationalen Argumenten gegen sie vorgegangen wird, was aber sehr selten geschieht. Valian erklärt in ihrem Buch psychologisch und soziologisch genau das, was das Frauen-Komitee am MIT als Fakten dokumentiert hat. Valian empfiehlt Gegenmaßnahmen, in deren Anwendung sich jedermann - Frau und Mann - trainieren kann wie z.B. kleine Nachteile nicht zu entschuldigen, sondern aufzudecken, anzusprechen und zu korrigieren. Wird das Bewusstsein jedoch erst einmal für diese Unterschiede geschärft, so wird man feststellen, dass der Arztalltag überfüllt ist mit derlei Begebenheit, wie ein kleines Beispiel verdeutlicht.

Während einer Veranstaltung einer pharmazeutischen Firma sitzen die männlichen Experten auf dem Podium, gut plaziert und mit grossen Namensschildern versehen. Einer dieser Experten hält weder einen Vortrag noch trägt er zur Diskussion bei, sondern sitzt schweigend auf dem Podium. Es gibt eine weibliche Expertin, die einen Vortrag hält. Dieser wird jedoch ständig von einem der männlichen Experten unterbrochen, wobei dieser jeweils ihre Worte bestätigt. Die weibliche Expertin hat keinen Sitzplatz auf dem Podium, sondern sitzt neben dem Diaprojektor im Auditorium, um die Dias der Kollegen zu schieben. Während der Diskussion bringt im Auditorium eine weibliche Chefärztin eine berechtigte kritische Bemerkung an. Ein ungeduldiger Kommentar eines männlichen Chefarztes, dass das "Mädel erst mal richtig nachdenken soll, bevor es den Mund aufmacht", bleibt unwidersprochen, es wird nicht protestiert. Dies dokumentiert, dass diese Ungleichbehandlungen gar nicht auffallen, wenn die Sinne nicht dafür geschärft sind. Den Menschen ist nicht bewusst, dass sie nach nicht mehr zeitgemäßen Geschlechterschemata handeln.

Noch hat es am MIT keinen weiblichen Dekan, keinen weiblichen Abteilungsleiter oder weibliche Vertreter gegeben. Bei uns in Deutschland stellen weibliche Professoren, Dekane, Rektoren, Chefärzte und Oberärzte im Jahre 2000 Ausnahmen dar. Die in Tab.1 aufgeführten Empfehlungen an die Verwaltung des MIT können auf jede Universität, Forschungseinrichtung oder Krankenhaus übertragen werden und eine positive Entwicklung bewirken. Erst wenn es einmal selbstverständlich sein wird, dass Frauen leitende Positionen einnehmen, wenn korrigierte Geschlechterschemata internalisiert wurden, erst dann wird das Geschlechterproblem wirklich gelöst sein - in der Wissenschaft, der akademischen Medizin, der praktischen Medizin genauso wie im Alltag.

\section{Literatur}

1 A study on the status of women faculty in science at MIT. MIT Faculty Newsletter, Vol. 11, No. 4. Cambridge, Mass, Massachusetts Institute of Technology (http://web.mit.edu/fnl/women/women.html), 1999

2 Bulmahn E. Women in science in Germany. Science 1999; 286: 2081

3 Lawler A. Tenured women battle to make it less lonely at the top. Science 1999; 286: 1272-1279

4 National Science Foundation, 1996. Characteristics of doctoral students and engineers in the United States: 1993. In: Valian V, editor. Why so slow? The advancement of women. MIT Press, 1999: 224

5 Statistisches Bundesamt Wiesbaden. Statistisches Jahrbuch. Verlag Metzler-Poeschel, Wiesbaden, 1981-1999

6 Valian V. Why so slow? The advancement of women. MIT Press, Cambridge, Massachusetts, 1999

Priv.-Doz. Dr. Dr. Margit Zuber

Institut für Klinische Immunologie und Transfusionsmedizin der Universität Leipzig

Haertelstrasse 16-18

04107 Leipzig

Tel.: 0341/9725538

Fax: 0341/9725569

E-Mail: zubm@medizin.uni-leipzig.de 